\title{
Pay-as-you-go Social Security and the Distribution of Bequests*
}

\author{
Jordi Caballé ${ }^{\dagger} \quad$ Luisa Fuster ${ }^{\ddagger}$
}

February 2000

\footnotetext{
* Financial support from the Spanish Ministry of Education through grants PB96-1160-C02-02 and SEC98-0301 and from the Generalitat of Catalonia through grant SGR98-00062 is gratefully acknowledged.

† Unitat de Fonaments de l'Anàlisi Econòmica and CODE, Universitat Autònoma de Barcelona.

$\ddagger$ Departament d’Economia i Empresa, Universitat Pompeu Fabra and Department of Economics, University of Western Ontario.
} 
Pay-as-you-go Social Security and the Distribution of Bequests

\begin{abstract}
This paper studies the impact of an unfunded social security system on the distribution of bequests in a framework where savings are due both by life cycle and by random altruistic motivations. We show that the impact of social security on the distribution of bequests depends crucially on the importance of the bequest motive in explaining savings behavior. If the bequest motive is strong, then an increase in the social security tax raises the bequests left by altruistic parents. On the other hand, when the importance of bequests in motivating savings is sufficiently low, the increase in the social security tax could result in a reduction of the bequests left by altruistic parents under some conditions on the attitude of individuals toward risk and on the relative returns associated with private saving and social security. Some implications concerning the transitional effects of introducing an unfunded social security scheme are also discussed.
\end{abstract}




\section{Introduction}

The effects of the social security system both on capital accumulation and on wealth distribution have received a great deal of attention among economists. Since most studies have considered life cycle economies with non-altruistic agents, the effects of social security on inheritances have been mostly neglected. This seems an important omission since there is substantial evidence that intergenerational transfers are crucial for understanding capital accumulation and wealth distribution in the US economy (see, for instance, Kotlikoff and Summers, 1981). This paper studies the impact of an unfunded social security system on the distribution of inheritances in a framework where savings are due both by life cycle and by random altruistic motivations. We show that the impact of social security on that distribution depends crucially on the importance of the bequest motive in explaining savings behavior. If the bequest motive is strong, then an increase in the social security tax raises the bequests left by altruistic parents. On the other hand, when the importance of bequests in motivating savings is sufficiently low, the increase in the social security tax could result in a reduction of the bequests left by altruistic parents under some conditions.

This paper develops a model where individuals save for altruistic and life-cycle motives that is simple enough to study distributional issues analytically. Individuals will live for two periods and they will work only during the first period. When individuals are young, they save to finance their consumption during the second period of their life. Altruism is modeled as a uninsurable random shock on preferences as in Escolano (1992) and Dutta and Michel (1998) among others. However, in the latter paper individuals are assumed to live only for one period and all the savings arise from the bequest motive. Of course, by not allowing the coexistence of at least two generations in the same period, such a framework is not suitable to analyze 
the effects of the inter-vivos transfer implicit in the pay-as-you-go (PAYG) social security system. In our model individuals face an idiosyncratic shock that determines whether they love their children or not. The shock on altruism implies in fact that the intertemporal discount rate of utility is a random variable. Individuals will save to finance both their second period consumption and the bequests in case they turn out to be altruistic.

We characterize the distribution of bequests at the steady state equilibrium and study how this distribution changes with the social security tax. In this economy, the bequest motive is operative for altruistic agents depending on the values of both the interest and the discount rate. If the bequest motive is not operative at the steady state equilibrium, social security does not affect the long run distribution of bequests since this distribution remains degenerate at zero. However, when the bequest motive is operative, social security affects the long run distribution of bequests. Whether it increases or decreases the size of altruistic bequests depends on the strength of the bequest motive as measured by the probability of being altruistic and on the return of social security relative to the return of capital.

We find that bequests increase with the size of pensions when the bequest motive is operative in the steady state and the probability of being altruistic is sufficiently high. Since an increase in pensions imposes higher mandatory transfers from the young to the old, altruistic parents find optimal to increase the size of the bequests they leave to their children. In the limit case of a probability of being altruistic equal to one, the increase in bequests completely undo the intergenerational transfers imposed by the PAYG social security system, as it was shown by Barro (1974). Therefore, when only a small subset of individuals are non-altruistic, a higher pension tax sparks off an increase of the wealth gap between children born in selfish families and children born in altruistic households.

On the contrary, bequests can decrease with pension benefits when the probability of being altruistic is sufficiently low. More precisely, we prove that, if preferences exhibit decreasing absolute risk aversion and the return from saving is greater than 
the return of the social security system (which is given by the rate of population growth), such a decrease of bequests takes place and, thus, the wealth gap between children born in selfish families and children born in altruistic ones becomes smaller.

Another result of our paper concerns the transitional effects of social security. As we have said, the altruistic individuals of the economy could decide not to leave bequests in the steady sate, that is, the bequests motive could be non-operative under some parametric restrictions (see Weil, 1987). In this case, the introduction of an unfunded social security system could force altruistic individuals to leave some bequests so as to absorb the initial impact of the mandatory transfers inherent in the social security system. Of course, non-altruistic individuals will remain leaving zero bequests. Therefore, the introduction of social security could induce a transitional dynamics characterized by inequality in the distribution of wealth at birth. Such effect is just transitory since the bequests of all individuals will converge to zero in the long run.

Among the papers analyzing the impact of fiscal policies on the distribution of bequests, we mention the ones of Bevan and Stiglitz (1979), Becker and Tomes (1979), Atkinson (1980), and Davies (1986). These authors focus on intragenerational redistributive policies and their effects on wealth and income distribution. Our paper contributes to this literature with an analytical study on the effects of a PAYG social security system on the distribution of altruistic bequests. Abel (1985) analyzes instead the impact of social security in a framework where bequests are accidental and only arise because of life time uncertainty. ${ }^{1}$ He finds that social security reduces accidental bequests because it annuitizes the wealth of individuals. In his model the fraction of individual savings made compulsory by the social security is returned as a pension only if the corresponding individual survives. Therefore, thanks to this public provision of annuities, individuals will make less voluntary savings and thus accidental bequests will be smaller. Obviously, this results in a reduction of

\footnotetext{
${ }^{1}$ Other papers analyzing different effects of social security under uncertain lifetimes are the ones of Eckstein et al. (1985) and Sheshinsky and Weiss (1981).
} 
the intracohort variance of wealth. Our approach consists instead on characterizing the impact of PAYG social security on the distribution of bequests when these are not accidental but altruistically motivated. In contrast to the result obtained with accidental bequests, such an impact could be ambiguous in the long run when the bequest motive is operative.

On a related paper Karni and Zilcha (1989) examine the effects of social security on income distribution. They emphasize the fact that, due to general equilibrium effects, social security induces a decrease of the return of labor relative to the return of capital. This effect leads to an increase of income inequality in their model because the only source of heterogeneity is an exogenous distribution of bequests (or initial capital). On the contrary, our paper focuses on the effect of social security on the distribution of bequests, which is not longer viewed as exogenous.

Finally, we should point out that random altruism can be interpreted as a shock on the intertemporal discount rate of utility and that recently some authors have used models with stochastic discount factors (see, for instance, Krusell and Smith (1998)). Random discounting has proven a useful device for generating wealth heterogeneity in quantitative models.

The paper is organized as follows. The next section describes the economic environment and the individuals' problem. Section 3 studies the dynamics of bequests and the operativeness of the bequest motive both in the short and in the long run. Section 4 shows the existence and uniqueness of the invariant distribution of bequests and characterizes this distribution. Section 5 focuses on the effects of social security on the distribution of bequests when the bequest motive is not operative in a steady state, whereas Section 6 performs the comparative statics analysis for economies where the bequest motive is always operative for altruistic agents. Section 7 concludes the paper. 


\section{The Model}

Let us consider an overlapping generations economy in discrete time with a continuum of individuals in each period. A generation of individuals with identical ex-ante preferences is born in each period and individuals live for two periods. At the end of the first period of his life each agent has $N \geq 1$ children so that an individual is young when his parent is old.

When individuals become old, they realize if they love their children, that is, they know if they are altruistic or not. If an individual is altruistic, the indirect utility of each of his children appears as an argument in his utility function. That event occurs with probability $\pi \in(0,1)$. For the sake of simplicity, we will assume that the total utility obtained by an altruistic old individual is the sum of the utility derived from his consumption when old and the sum of the indirect utilities of their direct descendants. Old individuals are selfish with probability $1-\pi$ and, then, they derive utility only from their own consumption. Therefore, in this large economy a mass $\pi$ of individuals is altruistic whereas a mass $1-\pi$ turns to be selfish. There are no markets to buy insurance against the risk of becoming altruistic towards children.

We will assume that the economy under consideration is an small open one with perfect capital mobility and where there is no labor mobility. This means that the interest rate is constant and equal to its international level. Let $R>0$ be the constant one-period gross rate of return on saving. The technology of this economy is represented by a production function with two inputs: labor and capital. Such a production function is strictly increasing, concave, and exhibits constant returns to scale. Both capital and labor markets are hired in perfectly competitive markets so that the rental prices of both inputs coincide with their respective marginal productivities. Therefore, given a fixed international interest rate, the capital-labor ratio is constant and, thus, the marginal productivity of labor is also constant. Such a marginal productivity of labor is in turn equal to the real wage $w$ in equilibrium. Young individuals are endowed with a unit of labor time. They supply their labor 
endowment inelastically in exchange for the constant market wage $w$. Old individuals are retired.

There is a government that administrates a balanced PAYG social security system. Young individuals contribute to the system by paying a constant lumpsum tax $P$ with $0 \leq P<w$. Therefore, each old individual receives a pension benefit equal to $N P$.

The utility that an individual derives from his own consumption in each period is represented by a utility function $u$ defined on $\mathbb{R}_{++}$which is assumed to be bounded and twice continuously differentiable with $u^{\prime}>0, u^{\prime \prime}<0$ and to satisfy the Inada conditions $\lim _{c \rightarrow 0} u^{\prime}(c)=\infty$ and $\lim _{c \rightarrow \infty} u^{\prime}(c)=0 .^{2}$

Young individuals only differ in the bequests they receive from their respective parents. A young individual that has received the bequest $b$ from his parent solves the following stochastic dynamic programming problem when the pension is fixed at the level $P$ :

$$
V_{y}(b ; P)=\underset{\left\{c_{y}, c_{s}, s\right\}}{\operatorname{Max}} u\left(c_{y}\right)+\beta\left\{(1-\pi) u\left(c_{s}\right)+\pi V_{a}(s ; P)\right\}
$$

subject to

$$
\begin{gathered}
c_{y}=w+b-P-s \geq 0, \\
c_{s}=R s+N P \geq 0,
\end{gathered}
$$

where $c_{y}$ is the consumption of a young individual, $s$ is the saving, $c_{s}$ is the

\footnotetext{
${ }^{1}$ When labor supply is inelastic, lump-sum social security taxes are equivalent to proportional taxes on wages since there is no distortion in the labor market.

${ }^{2}$ Instead of assuming that $u$ is bounded we could assume specific functional forms that are not bounded but that are quite common in the literature. For instance, if we assume isoelastic preferences, $u(c)=\frac{c^{1-\sigma}}{1-\sigma}$ with $\sigma>0$, then all the results of this paper are still true when the condition $\beta N \pi R^{1-\sigma}<1$ for bounded value functions holds. If $u(c)=\ln c$, then the corresponding condition is simply $\beta N \pi<1$.

Another commonly used utility function is the CARA $u(c)=-e^{-\gamma c}$ with $\gamma>0$. All the main results of the paper apply for this function in spite of not satisfying the Inada condition at the origin.
} 
consumption of a selfish old individual, $V_{y}(b ; P)$ is the value function of a young individual who has received a bequest $b$ when the level of pensions is $P$, and $V_{a}(s ; P)$ is the value function of an altruistic old individual who has saved the amount $s$ in his first period of life when the level of pensions is $P$. Note that the two weak inequalities in the constraints imply that $b \geq-w+P\left(\frac{R-N}{R}\right)$, where $-w+P\left(\frac{R-N}{R}\right)<0$ since $P \in[0, w)$. The coefficient $\beta>0$ is the time discount factor.

An old altruistic agent who saved the amount $s$ when he was young solves the following problem:

$$
V_{a}(s ; P)=\underset{\left\{c_{a}, b^{\prime}\right\}}{\operatorname{Max}} u\left(c_{a}\right)+N V_{y}\left(b^{\prime} ; P\right),
$$

subject to

$$
\begin{gathered}
c_{a}=R s+N P-N b^{\prime} \geq 0, \\
b^{\prime} \geq 0
\end{gathered}
$$

where $c_{a}$ is the consumption of an altruistic old individual and $b^{\prime}$ is the bequest that he leaves to each of his children, respectively. We could have assumed instead that the coefficient of the value function $V_{y}$ be any function of the number $N$ of direct descendants. None of our results would be qualitatively affected by this alternative assumption since the fertility rate is exogenous. Note that, since in this model altruism is unidirectional (it goes from parents to children) and there are neither institutions nor contracts to enforce liabilities on future generations, individuals cannot leave negative bequests to their children.

Plugging program (2.1) on program (2.2) and using the corresponding budget constraints, we obtain the following stochastic dynamic programming problem:

$$
\begin{aligned}
& \qquad V_{a}(s ; P)= \\
& \underset{\left\{b^{\prime}, s^{\prime}\right\}}{\operatorname{aax}} u\left(R s+N P-N b^{\prime}\right)+N u\left(w-P+b^{\prime}-s^{\prime}\right)+\beta N(1-\pi) u\left(R s^{\prime}+N P\right)+\beta N \pi V_{a}\left(s^{\prime} ; P\right) \\
& \text { subject to }
\end{aligned}
$$

$$
R s+P \geq N b^{\prime} \geq 0
$$




$$
w-P+b^{\prime} \geq s^{\prime} \geq-\frac{N P}{R},
$$

where $s^{\prime}$ is the saving of each descendant of the agent under consideration. ${ }^{3}$ Note that the Inada conditions on the utility function imply that $c_{y}, c_{a}$, and $c_{s}$ are all strictly positive so that the solution of the program (2.3) must satisfy $w-P+b^{\prime}>s^{\prime}>-\frac{N P}{R}$ and $R s+N P>N b^{\prime}$. Of course, it remains the possibility of a corner solution for bequests, namely, that $b^{\prime}=0$. Moreover, in order to have a well defined problem, we need that $s>-\frac{N P}{R}$ since neither $c_{a}$ nor $c_{s}$ could be positive otherwise. Finally, we assume that $\beta N \pi<1$.

Let us define the policy functions corresponding to the previous programs when the pension level is $P$. Concerning program (2.1), we define the policy functions $c_{y}=\hat{c}_{y}(b ; P), c_{s}=\hat{c}_{s}(b ; P)$, and $s=\hat{s}(b ; P)$ for consumption of a young individual, consumption of a selfish old individual, and saving, respectively. For program (2.2) we define $c_{a}=\hat{c}_{a}(s ; P)$ and $b^{\prime}=\hat{b}(s ; P)$ as the policy functions for consumption of an altruistic old individual and bequest per capita, respectively. We also define the composite function $g(b ; P) \equiv \hat{b}(\hat{s}(b ; P) ; P)$ which gives the bequest per capita left by an old altruistic individual who had received the transfer $b$ from his parent when the pension remained unchanged at the level $P$.

The following lemma establishes a basic result about the existence of a unique solution to the previous programs. Its proof is omitted since it follows immediately from applying, for instance, Theorems 4.6-4.10 of Stokey and Lucas (1989) to program (2.3). The existence and the properties of the value function $V_{y}(\cdot ; P)$ follow directly from the existence and properties of the value function $V_{a}(\cdot ; P)$ through program $(2.1)$.

Lemma 1. There exists a unique value function $V_{a}(\cdot ; P)$ associated with program (2.3) which is continuously differentiable, strictly increasing, and strictly concave

\footnotetext{
${ }^{3}$ We are implicitly assuming in problem (2.3) that parents choose the amount of saving $s^{\prime}$ of their descendants. This can be safely assumed since the sequentiality of the problem ensures that the saving of each son depends exclussively on the bequest he receives. Such a sequentiality prevents any kind of strategic behavior between parents and children.
} 
on $\left(-\frac{N P}{R}, \infty\right)$. The value function $V_{y}(\cdot ; P)$ exists and is continuously differentiable, strictly increasing and strictly concave on $\left(-w+P\left(\frac{R-N}{R}\right), \infty\right)$. The programs (2.1) and (2.2) have a unique solution, that is, the policy functions $\hat{c}_{y}(\cdot ; P), \hat{c}_{s}(\cdot ; P)$, $\hat{c}_{a}(\cdot ; P), \hat{b}(\cdot ; P)$, and $\hat{s}(\cdot ; P)$ exist. The functions $\hat{c}_{y}(\cdot ; P), \hat{c}_{s}(\cdot ; P), \hat{s}(\cdot ; P)$ and $g(\cdot ; P)$ are all continuous on $\left(-w+P\left(\frac{R-N}{R}\right), \infty\right)$, whereas the functions $\hat{c}_{a}(\cdot ; P)$ and $\hat{b}(\cdot ; P)$ are continuous on $\left(-\frac{N P}{R}, \infty\right)$.

Substituting the consumptions in the objective function of program (2.1) and differentiating with respect to $s$, we obtain the following first order condition:

$$
u^{\prime}(w+b-P-s)=\beta\left\{(1-\pi) R u^{\prime}(R s+N P)+\pi V_{a}^{\prime}(s ; P)\right\}
$$

and the corresponding envelope condition is

$$
V_{y}^{\prime}(b ; P)=u^{\prime}(w+b-P-s) .
$$

Concerning program (2.2), we substitute the consumption $c_{a}$ into the objective function and differentiate with respect to $b^{\prime}$ to obtain the following first order condition:

$$
u^{\prime}\left(R s+N P-N b^{\prime}\right) \geq V_{y}^{\prime}\left(b^{\prime} ; P\right), \text { and } u^{\prime}\left(R s+N P-N b^{\prime}\right)=V_{y}^{\prime}\left(b^{\prime} ; P\right) \text { if } b^{\prime}>0,
$$

and the corresponding envelope condition is

$$
V_{a}^{\prime}(s ; P)=R u^{\prime}\left(R s+N P-N b^{\prime}\right) .
$$

The following lemma provides additional properties of the policy functions:

Lemma 2. The policy functions $\hat{c}_{y}(\cdot ; P), \hat{c}_{s}(\cdot ; P), \hat{c}_{a}(\cdot ; P), \hat{s}(\cdot ; P)$ are all strictly increasing. The policy function $\hat{b}(\cdot ; P)$ is non-decreasing and locally strictly increasing when $\hat{b}(s ; P)>0$. Moreover, the function $g(\cdot ; P)$ is non-decreasing and locally strictly increasing when $g(b ; P)>0$.

Proof. (a) $\hat{s}(\cdot ; P)$ is strictly increasing. Let us proceed by contradiction. Let $b_{1}>b_{2}$ and assume that $s_{1} \leq s_{2}$ where $s_{1}=\hat{s}\left(b_{1} ; P\right)$ and $s_{2}=\hat{s}\left(b_{2} ; P\right)$, then $\beta\left\{(1-\pi) R u^{\prime}\left(R s_{1}+N P\right)+\pi V_{a}^{\prime}\left(s_{1} ; P\right)\right\} \geq \beta\left\{(1-\pi) R u^{\prime}\left(R s_{2}+N P\right)+\pi V_{a}^{\prime}\left(s_{2} ; P\right)\right\}$, 
as follows from the concavity of both $u$ and $V_{a}(\cdot ; P)$. From $(2.4)$ and the previous inequality, we obtain

$$
u^{\prime}\left(w+b_{1}-P-s_{1}\right) \geq u^{\prime}\left(w+b_{2}-P-s_{2}\right) .
$$

We get a contradiction by noticing that $b_{1}-s_{1}>b_{2}-s_{2}$, which is incompatible with the concavity of $u$.

(b) $\hat{c}_{s}(\cdot ; P)$ is strictly increasing. Obvious from part (a) and the fact that $\hat{c}_{s}(b ; P)=R \hat{s}(b ; P)+N P$.

(c) $\hat{c}_{y}(\cdot ; P)$ is strictly increasing. From the envelope condition $(2.5), V_{y}^{\prime}(b ; P)=$ $u^{\prime}\left(c_{y}(b ; P)\right)$, and the concavity of both $u$ and $V_{y}(\cdot ; P)$, the result immediately follows.

(d) $\hat{c}_{a}(\cdot ; P)$ is strictly increasing. From the envelope condition $(2.7), V_{a}^{\prime}(s ; P)=$ $u^{\prime}\left(c_{a}(s ; P)\right)$, and the concavity of both $u$ and $V_{a}(\cdot ; P)$, the result immediately follows.

(e) $\hat{b}(\cdot ; P)$ is non-decreasing and strictly increasing when $\hat{b}(s ; P)>0$. Assume that $s_{1}$ is an amount of saving for which $\hat{b}\left(s_{1} ; P\right)=0$. Then, for every $s_{2}>s_{1}$, we

have $\hat{b}\left(s_{2} ; P\right) \geq 0$ because of the non-negativity constraint on bequests. Therefore, $\hat{b}\left(s_{2} ; P\right) \geq \hat{b}\left(s_{1} ; P\right)$. On the other hand, assume that $s_{1}$ is such that $\hat{b}\left(s_{1} ; P\right)>0$. Then, condition (2.6) holds with equality and the concavity of both $u$ and $V_{y}(\cdot ; P)$ yields $\hat{b}\left(s_{2} ; P\right)>\hat{b}\left(s_{1} ; P\right)$ for every pair $s_{2}>s_{1}$.

(f) $g(\cdot ; P)$ is non-decreasing and locally strictly increasing when $g(b ; P)>0$. Obvious from (a) and (e).

\section{The Dynamics of Bequests within a Dynasty}

In this section we provide more properties of the function $g(\cdot ; P)$ defining the bequest left by an altruistic individual as a function of the transfer he has received from his parent under a stationary pension system. Several cases arise depending on both the discount factor $\beta$ and the gross rate of return from saving $R$.

The next proposition shows that, when the real interest rate $R-1$ is lower than the discount rate $\frac{1}{\beta}-1$ of utility, the sequence of inter-vivos transfers is strictly 
decreasing if all the members of a dynasty turn to be altruistic. Such a sequence of transfers converges asymptotically to zero.

Proposition 1. Assume that $R \beta \leq 1$. Then $g(b ; P)<b$ for all $b>0$ and $g(0 ; P)=0$.

Proof. Let $b>0$ be such that $b^{\prime}=g(b ; P)>0$. Then, we have

$$
\begin{aligned}
& u^{\prime}\left(\hat{c}_{y}(b ; P)\right)=R \beta\left\{(1-\pi) u^{\prime}\left(\hat{c}_{s}(b ; P)\right)+\pi u^{\prime}\left(\hat{c}_{a}(\hat{s}(b ; P) ; P)\right)\right\}< \\
& R \beta u^{\prime}\left(c_{a}(\hat{s}(b ; P) ; P)\right) \leq u^{\prime}\left(\hat{c}_{a}(\hat{s}(b ; P) ; P)\right)=V_{y}^{\prime}(g(b ; P) ; P)=u^{\prime}\left(\hat{c}_{y}(g(b ; P) ; P),\right.
\end{aligned}
$$

where the first equality comes from substituting the envelope condition (2.7) into the first order condition (2.4), the strict inequality comes from the fact that $\hat{c}_{a}(\hat{s}(b ; P) ; P)<\hat{c}_{s}(b ; P)$ when $b^{\prime}>0$, the weak inequality comes from the assumption that $R \beta \leq 1$, the second equality is just the first order condition (2.6) when $b^{\prime}>0$, whereas the last equality is the envelope condition $(2.5)$. Since $\hat{c}_{y}(\cdot ; P)$ is strictly increasing and $u$ is strictly concave, we get that $g(b ; P)<b$.

If $g(b ; P)=0$ for $b>0$, then it trivially follows that $g(b ; P)<b$.

Finally, let $b=0$. Since $g(b ; P)<b$ for all $b>0$, the continuity of $g(\cdot ; P)$ on $\left(-w+P\left(\frac{R-N}{R}\right), \infty\right)$ implies that $\lim _{b \rightarrow 0} g(b ; P)=g(0 ; P) \leq 0$. Hence, the nonnegativity constraint on bequests allows us to conclude that $g(0 ; P)=0$.

The next proposition strengthens the previous one since it gives a sufficient condition for the transfers to become zero after a finite history of altruistic individuals within a dynasty. Such a sufficient condition is obtained by just making strict the weak inequality which was assumed in Proposition 1, that is, the interest rate should be strictly lower than the discount rate of utility.

Proposition 2. Assume that $R \beta<1$. Then there exists a threshold level of bequests $\underline{b}(P)>0$ such that $g(b ; P)=0$ if and only if $b \leq \underline{b}(P)$.

Proof. Notice that

$$
V_{y}^{\prime}(0 ; P)=u^{\prime}\left(c_{y}(0 ; P)\right)=
$$




$$
\beta\left\{(1-\pi) R u^{\prime}(R \hat{s}(0 ; P)+N P)+\pi V_{a}^{\prime}(\hat{s}(0 ; P) ; P)\right\},
$$

where the first equality is the envelope condition (2.5) while the second is the first order condition (2.4). From Proposition 1, we know that $g(0 ; P)=0$ so that the envelope condition (2.7) becomes

$$
V_{a}^{\prime}(\hat{s}(0 ; P) ; P)=R u^{\prime}(R \hat{s}(0 ; P)+N P) .
$$

Combining (3.1) and (3.2), and using the fact that $R \beta<1$, we get

$$
V_{y}^{\prime}(0 ; P)<u^{\prime}(R \hat{s}(0 ; P)+N P)
$$

Define $\underline{b}(P)$ implicitly by

$$
V_{y}^{\prime}(0 ; P)=u^{\prime}(R \hat{s}(\underline{b}(P) ; P)+N P) .
$$

Note that $\underline{b}(P)>0$ since $\hat{s}(\cdot ; P)$ is strictly increasing and $u$ is strictly concave. Therefore, the following weak inequality holds for all $b \in[0, \underline{b}(P)]$ :

$$
V_{y}^{\prime}(0 ; P) \leq u^{\prime}(R \hat{s}(b ; P)+N P)
$$

We can show next that $g(b ; P)=0$ for $b \in[0, \underline{b}(P)]$. We proceed by contradiction and assume instead that $g(b ; P)>0$. Since both $V_{y}(\cdot ; P)$ and $u$ are strictly concave, we have that

$$
V_{y}^{\prime}(0 ; P)>V_{y}^{\prime}(g(b ; P) ; P)
$$

and

$$
u^{\prime}(R \hat{s}(b ; P)+N P-N g(b ; P))>u^{\prime}(R \hat{s}(b ; P)+N P) .
$$

These two inequalities, together with (3.4), imply that

$$
V_{y}^{\prime}(g(b ; P) ; P)<u^{\prime}(R \hat{s}(b ; P)+N P-N g(b ; N P)),
$$

which according to the first order condition (2.6) implies that $g(b ; P)=0$, and this is the desired contradiction. 
To prove that $g(b ; P)>0$ for $b>\underline{b}(P)$ assume instead that $g(b ; P)=0$ to get a contradiction. Such a contradiction is easily obtained since $g(b ; P)=0$ implies that

$$
V_{a}^{\prime}(\hat{s}(0 ; P) ; P) \leq R u^{\prime}(R \hat{s}(b ; P)+N P),
$$

as dictated by the first order condition (2.6). On the other hand, $b>\underline{b}(P)$ implies that

$$
V_{a}^{\prime}(\hat{s}(0 ; P) ; P)>R u^{\prime}(R \hat{s}(b ; P)+N P),
$$

because of the definition of $\underline{b}(P)$ in $(3.2)$ and the monotonicity of $\hat{s}(\cdot ; P)$. We obtain thus the desired contradiction.

The next proposition shows that the bequest motive is always operative when both the interest rate and the discount factor are high enough.

Proposition 3. If $R \beta>1$, then $g(b ; P)>0$ for all $b \geq 0$.

Proof. We will prove it by contradiction. Assume that $g(b ; P)=0$, then the first order condition (2.4) and the envelope condition (2.7) imply that

$$
\begin{gathered}
u^{\prime}\left(\hat{c}_{y}(b ; P)\right)= \\
R \beta\left\{(1-\pi) u^{\prime}(R \hat{s}(b ; P)+N P)+\pi u^{\prime}(R \hat{s}(b ; P)+N P)\right\}>u^{\prime}(R \hat{s}(b ; P)+N P),
\end{gathered}
$$

where the inequality comes from the assumption that $R \beta>1$. Moreover, when $g(b ; P)=0$ we have

$$
u^{\prime}(R \hat{s}(b ; P)+N P) \geq V_{y}^{\prime}(0 ; P)=u^{\prime}\left(c_{y}(0 ; P)\right)
$$

where the weak inequality is the corresponding first order condition (2.6) and the equality is the envelope condition (2.5). Combining (3.6) with (3.7), we get

$$
u^{\prime}\left(\hat{c}_{y}(b ; P)\right)>u^{\prime}\left(\hat{c}_{y}(0 ; P)\right)
$$

¿From the concavity of $u$ and the fact that $\hat{c}_{y}(\cdot ; P)$ is increasing, it follows that $b<0$, which is the desired contradiction. 
The properties of the bequest function we have just described will be extensively used in the next section in order to explore the existence and the properties of the stationary distribution of bequests in this economy.

\section{The Distribution of Bequests}

The distribution of bequests across individuals in each period is a probability measure defined on the measurable space $\left(\mathbb{R}_{+}, \mathcal{B}\right)$ where $\mathcal{B}$ is the $\sigma$-algebra of Borel sets of $\mathbb{R}_{+}$. In this section we will show that, given a constant level $P$ of the pension, the probability measure of bequests converges to a unique invariant (or stationary) probability measure $\bar{\mu}(\cdot ; P)$ on $\left(\mathbb{R}_{+}, \mathcal{B}\right)$.

In this large economy a proportion $1-\pi$ of individuals receives a zero transfer from their parents while a proportion $\pi$ of individuals receives a transfer governed by the function $g(\cdot ; P)$. Clearly, if an individual has an altruistic parent who received the bequests $b_{t}$, then he will receive a bequest equal to $g\left(b_{t} ; P\right)$. Hence, the law of motion of bequests within a dynasty is the following:

$$
b_{t+1}= \begin{cases}0 & \text { with probability } 1-\pi, \\ g\left(b_{t} ; P\right) & \text { with probability } \pi .\end{cases}
$$

Therefore, the distribution of bequests $\mu_{t}(\cdot ; P)$ evolves along time as dictated by the following functional equation:

$$
\mu_{t+1}(B ; P)=(1-\pi) \mathcal{I}_{B}(0)+\pi \int_{g^{-1}(B ; P)} \mu_{t}(d b ; P),
$$

where $\mathcal{I}_{B}$ is the indicator function of the Borel set $B$ and

$$
g^{-1}(B ; P)=\{b \geq 0 \text { such that } g(b ; P) \in B\} .
$$

Proposition 4. There exists a unique probability measure $\bar{\mu}(\cdot ; P)$ on the measurable space $\left(\mathbb{R}_{+}, \mathcal{B}\right)$ such that, for every initial distribution of bequests $\mu_{0}(\cdot ; P)$, the sequence of distributions defined by equation (4.1) satisfies

$$
\lim _{t \rightarrow \infty}\left|\mu_{t}(B ; P)-\bar{\mu}(B ; P)\right|=0, \quad \text { for all } B \in \mathcal{B},
$$


and the convergence is uniform for all sets in $\mathcal{B}$.

Proof. Let $Q(b, B ; P)$ be the transition function of the Markov process of bequests when the pension is $P$. This transition function gives the probability that an individual receiving a bequest equal to $b$ leaves a bequest lying in the Borel set $B$. Therefore,

$$
Q(b, B ; P)=(1-\pi) \mathcal{I}_{B}(0)+\pi \mathcal{I}_{B}(g(b ; P)) .
$$

Let $B^{c}$ be the complementary of the Borel set $B$ in $\mathbb{R}_{+}$. It is obvious from (4.2) that for every $B \in \mathcal{B}, Q(b, B ; P) \geq 1-\pi$ if $0 \in B$, for all $b \in B$. Moreover, $Q\left(b, B^{c} ; P\right) \geq 1-\pi$ if $0 \in B^{c}$, for all $b \in B$. Note that this means that the Condition M in Section 11.4 of Stokey and Lucas (1989) holds and, therefore, from their Theorems 11.12 and 11.6 we get the desired uniform convergence result.

The next two propositions characterize the invariant distribution $\bar{\mu}(\cdot ; P)$ of bequests for different levels of both the interest and the discount rates. Note that such an invariant distribution satisfies

$$
\bar{\mu}(B ; P)=(1-\pi) \mathcal{I}_{B}(0)+\pi \int_{g^{-1}(B ; P)} \bar{\mu}(d b ; P),
$$

as follows from (4.1). We will show in the next two propositions that, if the interest rate is lower than the discount rate, the stationary distribution of bequests is degenerate and has unitary mass at zero. On the other hand, if the interest rate is higher than the intertemporal discount rate of utility, the invariant distribution of bequests is non-degenerate.

Proposition 5. If $R \beta \leq 1$, then the invariant distribution of bequests is degenerate at zero, $\bar{\mu}(\{0\} ; P)=1$. Moreover, if $R \beta<1$ then the convergence to this degenerate distribution is achieved in finite time, that is, there exists a $T^{*}>0$ such that, for all $t>T^{*}$,

$$
\mu_{t}(B ; P)=\bar{\mu}(B ; P), \quad \text { for all } B \in \mathcal{B}
$$


Proof. We will show that the distribution $\bar{\mu}(\{0\} ; P)=1$ satisfies the functional equation (4.3), that is,

$\bar{\mu}(\{0\} ; P)=(1-\pi) \mathcal{I}_{\{0\}}(0)+\pi \int_{g^{-1}(\{0\} ; P)} \bar{\mu}(d b ; P)=1-\pi+\pi \int_{g^{-1}(\{0\} ; P)} \bar{\mu}(d b ; P)$.

Observe that Proposition 1 applies and $\{0\} \in g^{-1}(\{0\} ; P)$ since $R \beta \leq 1$. Therefore,

$$
\pi \int_{g^{-1}(\{0\} ; P)} \bar{\mu}(d b ; P)=\pi
$$

The last part of the proposition follows immediately from Propositions 1 and 2.

Proposition 6. If $R \beta>1$, then the distribution of bequests is non-degenerate and is given by the probability measure satisfying $\bar{\mu}\left(\left\{b_{i}\right\} ; P\right)=(1-\pi) \pi^{i}$ for $i=0,1, \ldots$, where $b_{i+1}=g\left(b_{i} ; P\right)$ and $b_{0}=0$.

Proof. We will show that the distribution in the statement satisfies (4.3). If $b_{i}>0$, we have from Proposition 3 that $b_{i+1}=g\left(b_{i} ; P\right)>0$. Then, since $g(\cdot ; P)$ is strictly increasing, as it is established by Lemma $2, g^{-1}\left(\left\{b_{i+1}\right\} ; P\right)=b_{i}$ and equation (4.3) becomes

$$
\bar{\mu}\left(\left\{b_{i+1}\right\} ; P\right)=(1-\pi) \mathcal{I}_{\left\{b_{i+1}\right\}}(0)+\pi \int_{b_{i}} \bar{\mu}(d b ; P)=0+\pi \bar{\mu}\left(\left\{b_{i}\right\} ; P\right)=(1-\pi) \pi^{i+1}
$$

since $\bar{\mu}\left(\left\{b_{i}\right\} ; P\right)=(1-\pi) \pi^{i}$. On the other hand, if $b_{i}=0$, then

$$
\bar{\mu}(\{0\} ; P)=(1-\pi) \mathcal{I}_{\{0\}}(0)+\pi \int_{g^{-1}(\{0\} ; P)} \bar{\mu}(d b ; P)=1-\pi,
$$

since Proposition 3 implies that the set $g^{-1}(\{0\} ; P)$ is empty and, thus, has zero measure.

Clearly, when $R \beta>1$ the average bequest under the stationary distribution is $(1-\pi) \sum_{0}^{\infty} \pi^{i} b_{i}$, where $b_{i+1}=g\left(b_{i} ; P\right)$ and $b_{0}=0$. Concerning the support of the stationary distribution $\bar{\mu}(\cdot ; P)$, the next proposition and corollaries establish that it can be either bounded or unbounded depending on the gross return on savings. 
Proposition 7. For given values of $\pi, \beta$, and $R$ such that $R \beta>1$, the support of the non-degenerate stationary distribution $\bar{\mu}(\cdot ; P)$ is bounded if and only if there exists a strictly positive solution for $b$ to the following equation:

$$
\frac{1-\pi \beta R}{\beta R(1-\pi)}=h(b)
$$

where

$$
h(b)=\frac{u^{\prime}\left(\left(\frac{R}{1+R}\right) w+\left(\frac{N-R}{1+R}\right) P+\left(\frac{R(1+N)}{1+R}\right) b\right)}{u^{\prime}\left(\left(\frac{R}{1+R}\right) w+\left(\frac{N-R}{1+R}\right) P-\left(\frac{N-R}{1+R}\right) b\right)} .
$$

Proof. Since $R \beta>1$ the bequest distribution is non-degenerate. Moreover, if the function $g(b ; P)$ has at least a strictly positive fixed point $b^{*}=g\left(b^{*} ; P\right)$, it follows that $\hat{s}\left(b^{*} ; P\right)=\hat{s}\left(g\left(b^{*} ; P\right) ; P\right)$. Let us define $s^{*}=\hat{s}\left(b^{*} ; P\right)$. Clearly, $b^{*}$ and $s^{*}$ must be solutions for $b$ and $s$ to the following system of equations:

$$
u^{\prime}(w+b-P-s)=\beta R\left\{(1-\pi) u^{\prime}(R s+N P)+\pi u^{\prime}(R s+N P-N b\}\right.
$$

and

$$
u^{\prime}(R s+N P-N b)=u^{\prime}(w+b-P-s)
$$

Equation (4.6) comes first from combining the envelope condition (2.7) with the first order condition (2.4), while equation (4.7) comes from combining the envelope condition (2.5) with the first order condition (2.6) when the latter holds with equality since $R \beta>1$ implies that $b^{\prime}>0$. Moreover, to obtain equations (4.6) and (4.7) we impose $b^{\prime}=g(b ; P)=b$ and $s=\hat{s}(b ; P)=\hat{s}(g(b ; P) ; P)$. Note that the monotonicity of $u^{\prime}$ makes (4.7) equivalent to $R s+N P-N b=w+b-P-s$, which can be rewritten as

$$
s=\left(\frac{1}{1+R}\right)(w+(1+N) b-(1+N) P) .
$$

Plugging (4.8) into (4.6) and rearranging terms we get equation (4.4). Clearly, the strictly positive fixed point $b^{*}=g\left(b^{*} ; P\right)$ is the supremum of the support of $\bar{\mu}(\cdot ; P)$ since $\lim _{i \rightarrow \infty} g\left(b_{i} ; P\right)=b^{*}$ when $b_{i+1}=g\left(b_{i} ; P\right)$ and $b_{0}=0$, as dictated by Proposition 6. On the other hand, if equation (4.4) does not have a strictly positive solution for 
$b$, then the function $g(b ; P)$ does not have a fixed point so that $g(b ; P)>b$ for all $b \geq 0$ and, hence, $\lim _{i \rightarrow \infty} g\left(b_{i} ; P\right)=\infty$ when $b_{i+1}=g\left(b_{i} ; P\right)$ and $b_{0}=0$.

The next two corollaries provide sufficient conditions on the gross return of savings for the support of the stationary distribution $\bar{\mu}(\cdot ; P)$ of bequests to be either bounded or unbounded:

Corollary 1. The stationary distribution $\bar{\mu}(\cdot ; P)$ has bounded support whenever $R \leq \max \left\{N, \frac{1}{\beta}\right\}$.

Proof. We consider two cases: (i) If $R \leq \frac{1}{\beta}$, then the support of the distribution $\bar{\mu}(\cdot ; P)$ is obviously bounded since it is degenerate at zero, as follows from Proposition 5 .

(ii) Assume that $\frac{1}{\beta}<R \leq N$. In this case the stationary distribution of bequests is non-degenerate, and the function $h(b)$ defined in (4.5) is strictly decreasing since the strict concavity of the utility function implies that its numerator is strictly decreasing in $b$, whereas the denominator is non-decreasing in $b$. Note that $h(0)=1$. Moreover, if $R<N$,

$$
\lim _{b \rightarrow\left(\frac{R}{N-R}\right) w+P} h(b)=0,
$$

whereas if $R=N, \lim _{b \rightarrow \infty} h(b)=0$. On the other hand, the LHS of equation (4.4) is strictly positive since $R \leq N<\frac{1}{\beta \pi}$, and it is strictly smaller than 1 since $(1-\pi) \beta R \geq 1-\pi \beta R>0$ whenever $\frac{1}{\beta}<R$. Then, collecting all the previous facts, we conclude that equation (4.4) must have a unique, strictly positive solution for $b$.

Corollary 2. The stationary distribution $\bar{\mu}(\cdot ; P)$ has unbounded support whenever $R \geq \frac{1}{\beta \pi}$.

Proof. In this case, since $\pi \in(0,1)$, we have that $R \beta>1$ so that the bequest distribution is non-degenerate. Note that the LHS of equation (4.4) is non-positive 
when $R \geq \frac{1}{\beta \pi}$ and, since its RHS is always strictly positive for finite positive values of $b$, equation (4.4) has no finite, strictly positive solution for $b$. The result then follows from Proposition 7.

The previous corollaries tell us that, when the return $R$ from saving is lower than $N$, the wealth of a dynasty obviously decreases along time since the net return per capita from transferring wealth from one period to the following is always negative. On the other hand, a high interest rate allows a perpetually growing sequence of intervivos transfers within a dynasty formed exclusively by altruistic agents. Finally, note that these sufficient conditions for having either bounded or unbounded support are independent of the pension tax $P$.

We can illustrate the previous results with the following two examples:

Example 1. Assume that the utility function is CARA, $u(c)=-e^{-\gamma c}$ with $\gamma>0$. It is straightforward to see that, if $\frac{1}{\beta} \leq R<\frac{1}{\beta \pi}$, equation (4.4) has a strictly positive solution which is given by

$$
b^{*}=\frac{1}{N \gamma}\left[\ln \left(\frac{\beta R(1-\pi)}{1-\pi \beta R}\right)\right] .
$$

Note that the term inside the square brackets is well defined since $\beta R(1-\pi) \geq$ $1-\pi \beta R>0$ whenever $\frac{1}{\beta} \leq R<\frac{1}{\beta \pi}$. Hence, we can conclude that for the CARA utility the support of the distribution of bequests is bounded if and only if $R<\frac{1}{\beta \pi}$. This means that the sufficient condition given in Corollary 2 for unbounded support of the stationary distribution of bequests is also necessary when the utility function is CARA.

Example 2. Assume now logarithmic preferences, that is, $u(c)=\ln c$. In this case, after some algebra, it can be proved that equation (4.4) has a strictly positive solution for $b$ if and only if

$$
R<\frac{1+N(1+\beta(1-\pi))}{\beta(1+N \pi)} .
$$


Moreover, such a solution is unique and is given by

$$
b^{*}=\left(\frac{\beta R-1}{R}\right)\left(\frac{R(w-P)+N P}{1+N[1+\beta(1-\pi)]-\beta R(1+N \pi)}\right) .
$$

Note then that the sufficient conditions either for bounded or for unbounded support of $\bar{\mu}(\cdot ; P)$ given in the previous two corollaries are not necessary under logarithmic preferences. This is so because it can be checked that

$$
\max \left\{N, \frac{1}{\beta}\right\}<\frac{1+N(1+\beta(1-\pi))}{\beta(1+N \pi)}<\frac{1}{\beta \pi} .
$$

\section{Transitional Effects of Social Security when the Bequest Motive is Inoperative in the Long Run}

In this section we analyze how an unanticipated permanent introduction of a PAYG social security affects individuals' decisions and, thus, the distribution of bequests when the bequest motive is not operative in the long run, that is, when $R \beta \leq 1$. Note that, when the introduction of the social security scheme takes place, savings of an old agent are fixed and equal to $\hat{s}(0 ; 0)$, whereas the bequests he leaves if he becomes altruistic will be $\hat{b}(\hat{s}(0 ; 0) ; P)$, which is not necessarily equal to either $g(0 ; 0)=\hat{b}(\hat{s}(0 ; 0) ; 0)$ or $g(0 ; P)=\hat{b}(\hat{s}(0 ; P) ; P)$. Moreover, young individuals at the moment of the policy change will select their saving according to the function $\hat{s}(\cdot ; P)$.

In particular we will see that, when interest rates are low, there is a range of pension levels for which the unanticipated introduction of the social security system does not even affect the distribution of bequests in the short run so that it remains degenerate at zero. However, for higher values of the interest rate, there is a threshold level of pensions above which the introduction of the social security induces a non-degenerate distribution of bequests in the short run. Such a distribution will converge in the long run to the degenerate one in accordance with Propositions 4 and 5.

Proposition 8. Assume that $R \beta<1$ and that the initial distribution of bequests is the degenerate, invariant one (given in Proposition 5). There exists a pension $\operatorname{tax} \underline{P}$ such that, if the government introduces an unanticipated PAYG social 
security system with a pension tax level $P \in(0, \min \{w, \underline{P}\})$, then the bequest motive remains inoperative after the introduction of social security and, thus, the distribution of bequests remains degenerate at zero.

Proof. The stationary distribution of bequests when $P=0$ is degenerate at zero whenever $R<\frac{1}{\beta}$ as dictated by Proposition 5 . Let $\underline{P}=R[\hat{s}(\underline{b}(0) ; 0)-\hat{s}(0 ; 0)]$, where $\underline{b}(0)$ is defined in equation $(3.3)$. If $P<\min \{w, \underline{P}\}$, we get

$$
u^{\prime}(R \hat{s}(0 ; 0)+P)>u^{\prime}(R \hat{s}(0 ; 0)+\underline{P})=u^{\prime}(R \hat{s}(\underline{b}(0) ; 0))
$$

as a consequence of the strict concavity of $u$ and the definition of $\underline{P}$. From the definition of $\underline{b}(0)$ given in $(3.3)$, we have

$$
u^{\prime}(R \hat{s}(\underline{b}(0) ; 0))=V_{y}^{\prime}(0 ; 0)
$$

Combining (5.1) with (5.2) we obtain

$$
u^{\prime}(R \hat{s}(0 ; 0)+P)>V_{y}^{\prime}(0 ; P)
$$

which, from the first order condition $(2.6)$, implies that $\hat{b}(\hat{s}(0 ; 0) ; P)=0$ for all $P<$ $\min \{w, \underline{P}\}$. Therefore, the bequest motive remains inoperative after the introduction of this social security scheme and, thus, the degenerate initial distribution of bequests is not affected by such an introduction of pensions.

The previous proposition tell us that, in order to obtain a transitory effect on the distribution of bequests, we need that the introduction of the PAYG social security be sufficiently intense, that is, the pension tax $P$ should be sufficiently high. On the other hand, we will need an interest rate higher than the implicit rate of return of the social security system in order to prevent the present value of lifetime income of the descendants from increasing after the introduction of social security. Note in this respect that $w>w-P+\frac{N P}{R}$ if and only if $R>N$. In this situation, altruistic parents will react by returning part of the pension they receive to their descendants. However, the sons of non-altruistic parents will not enjoy such a compensation and 
this would give rise to a non-degenerate distribution of bequests and, thus, the PAYG system would be the source of some transitory inequality of wealth.

Before stating the precise result, we need to establish the following lemma:

Lemma 3. Let $P_{1}>P_{2}$ and assume that $g\left(b ; P_{1}\right)=g\left(b ; P_{2}\right)=0$, then

$$
\hat{s}\left(b ; P_{1}\right)<\hat{s}\left(b ; P_{2}\right) \quad \text { and } \quad \hat{c}_{y}\left(b ; P_{1}\right) \gtreqless \hat{c}_{y}\left(b ; P_{2}\right) \text { if } R<N
$$

Proof. Combine the first order condition (2.4) and the envelope condition (2.7) evaluated at $b^{\prime}=0$ to get

$$
u^{\prime}(w+b-P-s)=R \beta u^{\prime}(R s+N P) .
$$

Implicitly differentiating the previous equation, we obtain

$$
\frac{d s}{d P}=-\frac{u^{\prime \prime}(w+b-P-s)+R N \beta u^{\prime \prime}(R s+N P)}{u^{\prime \prime}(w+b-P-s)+R^{2} \beta u^{\prime \prime}(R s+N P)}<0 .
$$

It is straightforward to check that $\left|\frac{d s}{d P}\right| \gtrless 1$ if $R<N$. Since $c_{y}=w+b-P-s$, it follows that $\frac{d c_{y}}{d P} \gtrless 0$ if $R<N$.

Proposition 9. Assume that $N \leq R \leq 1 / \beta$ and that the initial distribution of bequests is the degenerate, invariant one (given in Proposition 5). There exists a pension $\bar{P} \geq 0$ such that the bequest motive becomes operative when the social security is introduced with a pension level $P \in(\bar{P}, w)$. In this case, the distribution of bequests becomes non-degenerate when social security is introduced and it converges back to the degenerate distribution.

Proof. Before introducing the social security scheme the bequests are zero and

$$
u^{\prime}(R \hat{s}(0 ; 0)) \geq u^{\prime}\left(\hat{c}_{y}(0 ; 0)\right)
$$

as follows from the condition (2.6) and the envelope condition (2.5) when $b^{\prime}=0$. Assume that $b^{\prime}=\hat{b}(\hat{s}(0 ; 0) ; P)=0$ and define the threshold pension $\bar{P}$ as the one that solves the following equation:

$$
u^{\prime}(R \hat{s}(0 ; 0)+\bar{P})=u^{\prime}\left(\hat{c}_{y}(0 ; \bar{P})\right)
$$


Such a threshold is non-negative since $u$ is strictly concave and $\hat{c}_{y}(0 ; P)$ is nonincreasing in $P$ for $R \geq N$ whenever $b^{\prime}=0$, as follows from Lemma 3. Moreover $\bar{P}<w$ as a consequence of the Inada conditions at the origin. Then,

$$
u^{\prime}(R \hat{s}(0 ; 0)+P)<u^{\prime}\left(\hat{c}_{y}(0 ; P)\right)
$$

for all $P>\bar{P}$, and this violates conditions (2.6) and (2.7). Therefore, the bequest motive should become operative $\left(b^{\prime}=\hat{b}(\hat{s}(0 ; 0) ; P)>0\right)$ after the introduction of a pension at a level higher than $\bar{P}$. Hence,

$$
u^{\prime}\left(R \hat{s}(0 ; 0)-b^{\prime}+P\right)=u^{\prime}\left(\hat{c}_{y}\left(b^{\prime} ; P\right)\right)
$$

since $u$ is strictly concave and $\hat{c}_{y}(\cdot ; P)$ is strictly monotonically increasing . Note that the saving of the agents who were old at the moment of the introduction of social security is given by $\hat{s}(0 ; 0)$. Finally, as it follows from Propositions 4 and 5 , the distribution of bequests after the introduction of the pension converges to the degenerate one since $\lim _{i \rightarrow \infty} g\left(b_{i} ; P\right)=0$ when $b_{i+1}=g\left(b_{i} ; P\right)$ and $b_{0}=0$.

According to the previous proposition the introduction of a permanent PAYG social security scheme could induce a non-degenerate distribution of bequests in the short run. Such an effect is transitory since bequests tend to zero in the long run even within dynasties displaying an infinite sequence of altruistic individuals when $R \beta<1$. Needless to say, the non-degenerate distribution of bequests in the short run is associated with a corresponding non-degenerate distribution of consumptions and savings through their respective policy functions.

\section{Long Run Effects of Social Security when the Bequest Motive is Always Operative}

In this section we will explore how a permanent marginal change in the social security tax affects both the bequest that individuals leave to their descendants and the long run distribution of bequest when the bequest motive is always operative for altruistic 
agents. This situation occurs when $R \beta>1$ and, thus, the stationary distribution of bequests is non-degenerate in this case.

If the bequest motive were non-random and always operative within a dynasty, then individuals would adjust their bequests in order to completely offset the change in the pension. In fact, individuals would only care about the net intergenerational transfer $b-P$ from parents to each descendant. This means that when $P$ increases, the bequest $b$ should increase by the same amount. However, if the altruism is random, individuals could react by decreasing their saving when the pension level increases. In fact, this is the typical reaction of non-altruistic individuals since they only care about the total saving $s+P$. As we will prove in this section, when the probability of being altruistic is low, then such a reduction in the amount of savings could encompass a reduction of the bequests left by the few individuals that turn to be altruistic.

The next proposition applies for economies populated basically by altruistic individuals.

Proposition 10. Let $R \beta>1$ and $P_{1}>P_{2}$. There exists a $\hat{\pi}>0$ such that $g\left(b ; P_{1}\right)>g\left(b ; P_{2}\right)$ for all $\pi \in(\hat{\pi}, 1)$. Moreover, $\lim _{\pi \rightarrow 1}\left(\frac{\partial g(b ; P)}{\partial P}\right)=1$.

Proof. For $\pi=1$ the result follows directly from Barro (1974) since the altruistic agents completely offset the effects of social security by means of adjustments in the amount of bequests within the dynasty when the bequests motive is always operative, i.e., when $R \beta>1$. In fact, from (2.5) and (2.6), we have that

$$
u^{\prime}\left(R \hat{s}\left(b ; P_{1}\right)-N g\left(b ; P_{1}\right)+N P_{1}\right)=u^{\prime}\left(w-P_{1}+g\left(b ; P_{1}\right)-\hat{s}\left(g\left(b ; P_{1}\right) ; P_{1}\right)\right),
$$

when the pensions were at level $P_{1}$ and the bequest motive is operative, whereas when pensions are set at level $P_{2}$ we have

$$
u^{\prime}\left(R \hat{s}\left(b ; P_{2}\right)-N g\left(b ; P_{2}\right)+N P_{2}\right)=u^{\prime}\left(w-P_{2}+g\left(b ; P_{2}\right)-\hat{s}\left(g\left(b ; P_{2}\right) ; P_{2}\right)\right) .
$$

Therefore, these two equations are compatible when $\hat{s}\left(b ; P_{1}\right)=\hat{s}\left(b ; P_{2}\right), g\left(b ; P_{1}\right)=$ 
$g\left(b ; P_{2}\right)+\left(P_{1}-P_{2}\right)$, and $\hat{s}\left(g\left(b ; P_{1}\right) ; P_{1}\right)=\hat{s}\left(g\left(b ; P_{2}\right) ; P_{2}\right)$. By continuity, if $\pi$ is close to 1 , then intergenerational transfers increase when social security is introduced.

Note that the implication of the previous proposition is that the stationary average bequest of this economy increases with the pension level when the probability $\pi$ of being altruistic is high enough and the altruistic agents leave positive bequests $(R \beta>1)$. Moreover, if we divide the population of the economy into two groups of individuals: the ones that receive bequests and the ones that do not, then all the values of the discrete support of the stationary distribution of bequests for the latter group suffer an increase. Obviously, the bequests for the former group remains unaltered at zero. This means that the expected wealth gap at birth between descendants of non-altruistic parents and descendants of altruistic ones increases with the pension in this scenario. A final obvious implication of the previous proposition is that the distribution of bequests associated to a lower pension tax is dominated by the distribution with a higher tax in the sense of first order stochastic dominance. This is so because the probability of having bequests lower than a given value $\bar{b}$ decreases as the pension $P$ increases for all $\bar{b}>0$ (see Hadar and Russell, 1969).

It is possible however to find examples in which the increase in the pension results in a reduction of the expected difference in wealth at birth between the two population groups we have just defined. The previous proposition tells us that, in order to find such examples, we need an operative bequest motive and, simultaneously, a low value of $\pi$. Moreover, if the individuals' preferences exhibit decreasing (increasing) absolute risk aversion (see Arrow, 1970, and Pratt, 1964), we will need to impose that the gross return $R$ from savings is higher (lower) than the gross rate $N$ of population growth. Note that, since individuals are non-altruistic with a very high probability, their decisions concerning their profile of consumption are mostly driven by the present value of their lifetime income as they abstract from bequests considerations. If $R>N$, the increase in the pension $P$ translates into a decrease in the present value of the lifetime income $\left(w+b-P+\frac{N P}{R}\right)$ of 
all agents. This in turn makes agents more (less) risk averse under decreasing (increasing) absolute risk aversion. Therefore, the few altruistic parents of this economy would react by decreasing (increasing) the bequest left to their sons. This is so because the increase (decrease) in risk aversion induces agents to decrease (increase) the difference between the old consumption corresponding to the event of being altruistic and the one corresponding to the event of being selfish. Of course, the opposite argument applies when $R<N$. The following two propositions formalize the previous discussion.

Proposition 11. Let $R \beta>1$ and $P>0$. There exists a $\pi^{*}>0$ such that $\frac{\partial g(b, P)}{\partial P}<0$ for all $\pi \in\left(0, \pi^{*}\right)$ whenever any of the following two conditions is satisfied:

(i) $R>N$ and the index of absolute risk aversion of $u, R_{A}=-u^{\prime \prime} / u^{\prime}$, is strictly decreasing.

(ii) $R<N$ and the index of absolute risk aversion of $u$ is strictly increasing.

Proof. See the appendix.

Even if the probability $\pi$ of being altruistic is very low, an increase in the pension could trigger an increase in the bequests left by altruistic agents. To get such a result we just need to assume the opposite to Proposition 11 and apply exactly the same reasoning.

Proposition 12. Let $R \beta>1$ and $P>0$. There exists a $\pi^{*}>0$ such that $\frac{\partial g(b, P)}{\partial P}>0$ for all $\pi \in\left(0, \pi^{*}\right)$ whenever any of the following two conditions is satisfied:

(i) $R<N$ and the index of absolute risk aversion of $u$ is strictly decreasing.

(ii) $R>N$ and the index of absolute risk aversion of $u$ is strictly increasing.

Proof. Obvious from the proof of Proposition 11.

Concerning the distribution of bequests (and, thus, of consumptions and savings), we see that, in the scenario depicted by the assumptions of Proposition 11, an increase in the pension $P$ results in a reduction of the bequests left by all the altruistic agents 
so that the expected wealth gap at birth between children of altruistic parents and children of egoist ones decreases with the pension level. Note that this is in sharp contrast to the situation where the probability of being altruistic was sufficiently high since there the increase in the pension level widened the gap of initial wealth between these two population groups. Moreover, under the assumptions of Proposition 11, the probability of having bequests lower than a given value $\bar{b}$ increases as the pension $P$ increases for all $\bar{b}>0$. Clearly, this means that the distribution of bequests with a lower social security tax dominates the one with a higher tax in the sense of first degree stochastic dominance.

In order to illustrate the previous two propositions, we can consider the Example 2 with logarithmic preferences and $\beta R>1$. Note that the upper bound $b^{*}$ given by (4.9) tends to

$$
\frac{\beta R-1}{R}\left(\frac{R(w-P)+N P}{1+N+(N-R) \beta}\right)
$$

as $\pi$ approaches zero. This limit is strictly positive whenever $1+N+(N-R) \beta>0$. Moreover, it is immediate to see that the limit of the upper bound $b^{*}$ is strictly decreasing (increasing) in $P$ when $R>(<) N$, which agrees with parts (i) of Propositions 11 and 12 since the logarithmic utility exhibits a strictly decreasing index of absolute risk aversion.

We conclude this section with some welfare considerations. It is obvious that the welfare of the generation that was old at the time when the pension increased was enhanced by such a policy change. On the other hand, if we were interested in the steady state effects on welfare, we should restrict our attention to the expected utility of the newborns under the stationary distribution of bequests. Note that, when the probability of being altruistic is very low, the welfare of individuals depends basically on the present value of their lifetime income. The effects of social security on the expected utility of newborns are generally ambiguous as it usually happens in OLG models. However, in the scenario depicted by part (i) of Proposition 11, we can say that the ex-ante welfare of a newborn is reduced since the present value of non- 
inherited lifetime income $\left(w-P+\frac{N P}{R}\right)$ decreases and the distribution of bequests becomes less desirable as a consequence of the shift in terms of first order stochastic dominance. The opposite holds in the scenario of part (i) of Proposition 12 and, thus, the ex-ante welfare of a newborn increases in such a case. Concerning parts (ii) of Propositions 11 and 12, the results are ambiguous since the present value of lifetime income and the shift on the bequests distribution have opposite effects on welfare.

\section{Conclusions}

In this paper we have characterized the distribution of bequests in an economy in which a PAYG social security system is present. We have analyzed both the transitory and the permanent effects on this distribution caused by changes in the pension tax. We have shown that the effects of social security on the distribution of bequests depends crucially on the importance of the bequest motive. If individuals are most likely altruists, then the introduction of social security increases the size of altruistic bequests. On the other hand, when individuals are most likely nonaltruistic, then the introduction of social security could reduce the those bequests under some conditions on the attitude of individuals toward risk and the relative returns associated with private saving and social security.

In contrast to our results, if the distribution of bequests were generated by uncertain lifetimes, then an increase in the pension tax would result unambiguously in an smaller wealth gap between the individuals who have received a positive bequests and the ones that have not. This is so because social security acts as a public annuity which reduces the size of accidental bequests (see Abel, 1985).

On the other hand, a non-degenerate distribution of bequests could also arise when there are two types of dynasties, the altruistic and the selfish ones (as in Michel and Pestieau, 1998). In this case individuals know when they are young if they are altruistic or not and, hence, they do not face any kind of risk. Obviously, 
an increase in the social security tax will always increase the bequest left by all the altruistic agents and this will also increase the wealth gap between the two types of dynasties.

We have focused on the unfunded social security system since, as we have seen, it is a system for which a quite rich plethora of results arises depending on the parametric assumptions of the model. If we had considered instead a fully funded social security system, the marginal changes in the social security tax would translate immediately into a change by the same amount in the voluntary savings. Therefore, the bequest left by an individual would remain unchanged since this bequest only depends on his effective saving regardless of whether it is compulsory or voluntary.

Finally, we point out that it could be interesting to characterize the distribution of bequests and the corresponding impact of social security if we introduced uncertainty on alternative sources of intended bequests. Among the different approaches, we mention the model of "joy-of-giving" in which parents derive direct utility from the size of the bequest they leave (Yaari, 1965) or the model of "strategic altruism" in which intergenerational transfers arise as payments for services provided by children (Bernheim et al., 1985). We leave this for future research. 


\section{A. Appendix}

Proof of Proposition 11. First, observe that, from (5.3), we get

$$
\lim _{\pi \rightarrow 0} \frac{d s}{d P}=-\frac{1+R \beta N Q}{1+R^{2} \beta Q}
$$

where $Q \equiv \frac{u^{\prime \prime}\left(\hat{c}_{s}(b ; P)\right)}{u^{\prime \prime}\left(\hat{c}_{y}(b ; P)\right)}$, and $\hat{c}_{s}(b ; P)=R s+N P$ and $\hat{c}_{y}(b ; p)=w+b-P-s$ satisfy

$$
u^{\prime}\left(\hat{c}_{y}(b ; P)\right)=R \beta u^{\prime}\left(\hat{c}_{s}(b ; P)\right),
$$

which is the limit of the first order condition of an altruistic individual when $\pi$ tends to zero. Note that the Inada conditions on the utility function $u$ prevents the limit of $V_{a}^{\prime}(s ; P)$ from tending to infinity. This is so because $V_{a}^{\prime}(s ; P)$ has to be equal to $R u^{\prime}\left(\hat{c}_{y}\left(b^{\prime} ; P\right)\right)$, as follows from combining $(2.5),(2.6)$ and $(2.7)$.

Combine now the conditions (2.4) and (2.7) corresponding to the maximization problem of a son of the individual under consideration to get

$$
\begin{aligned}
& u^{\prime}\left(w+b^{\prime}-P-s^{\prime}\right)= \\
& \quad R \beta\left\{(1-\pi) u^{\prime}\left(R s^{\prime}+N P\right)+\pi u^{\prime}\left(R s^{\prime}+N P-N b\left(s^{\prime} ; P\right)\right)\right\} .
\end{aligned}
$$

Implicit differentiation of $(A .3)$ yields the following limit

$$
\lim _{\pi \rightarrow 0} \frac{d b^{\prime}}{d P}=1+R \beta N Q^{\prime}+\left[1+R^{2} \beta Q^{\prime}\right]\left(\lim _{\pi \rightarrow 0} \frac{d s^{\prime}}{d P}\right),
$$

where $Q^{\prime} \equiv \frac{u^{\prime \prime}\left(\hat{c}_{s}\left(b^{\prime} ; P\right)\right)}{u^{\prime \prime}\left(\hat{c}_{y}\left(b^{\prime} ; P\right)\right)}$, and $\hat{c}_{s}\left(b^{\prime} ; P\right)=R s^{\prime}+N P$ and $\hat{c}_{y}\left(b^{\prime} ; p\right)=w+b^{\prime}-P-s^{\prime}$ satisfy

$$
u^{\prime}\left(\hat{c}_{y}\left(b^{\prime} ; P\right)\right)=R \beta u^{\prime}\left(\hat{c}_{s}\left(b^{\prime} ; P\right)\right) .
$$

Combine now the conditions (2.6) and (2.5) to get

$$
u^{\prime}\left(R s+N P-N b^{\prime}\right)=u^{\prime}\left(w+b^{\prime}-P-s^{\prime}\right) .
$$

Implicit differentiation of the previous equation yields

$$
\lim _{\pi \rightarrow 0} \frac{d s^{\prime}}{d P}=(1+N)\left(\lim _{\pi \rightarrow 0} \frac{d b^{\prime}}{d P}\right)-(1+N)-R\left(\lim _{\pi \rightarrow 0} \frac{d s}{d P}\right) .
$$


Combining $(A .4)$ and $(A .6)$, we get

$$
\left(\lim _{\pi \rightarrow 0} \frac{d b^{\prime}}{d P}\right)=\frac{R^{2} \beta Q^{\prime}-R \beta N Q^{\prime}+\left(1+R^{2} \beta Q^{\prime}\right)\left[N+R\left(\lim _{\pi \rightarrow 0} \frac{d s}{d P}\right)\right]}{N+R^{2} \beta Q^{\prime}(1+N)} .
$$

The sign of the above expression is the same as the one of its numerator. Note that we can rewrite $(A .5)$ as

$$
R \beta Q^{\prime}=\frac{R_{A}\left(\hat{c}_{s}\left(b^{\prime} ; P\right)\right)}{R_{A}\left(\hat{c}_{y}\left(b^{\prime} ; P\right)\right)},
$$

where $R_{A}(c)=\frac{-u^{\prime \prime}(c)}{u^{\prime}(c)}$ denotes the index of absolute risk aversion evaluated at $c$, and $\hat{c}_{s}\left(b^{\prime} ; P\right)$ and $\hat{c}_{y}\left(b^{\prime} ; p\right)$ satisfy the condition $(A .5)$. Analogously, we have that

$$
R \beta Q=\frac{R_{A}\left(\hat{c}_{s}(b ; P)\right)}{R_{A}\left(\hat{c}_{y}(b ; P)\right)} .
$$

We will proceed now with the proof of part (i). Assume thus that $R>N$ and that the coefficient of absolute risk aversion is decreasing. A decreasing coefficient of absolute risk aversion implies that $R_{A}\left(\hat{c}_{s}(b ; P)\right)<R_{A}\left(\hat{c}_{y}(b ; P)\right)$ because $\hat{c}_{s}(b ; P)>\hat{c}_{y}(b ; P)$ as follows from $(A .2)$ and the fact that $R \beta>1$. Hence, equation (A.9) implies that $R \beta Q<1$. Similarly, from $(A .5)$ and $(A .8)$, it holds that $R \beta Q^{\prime}<1$. From equation $(A .1)$ we get the following useful expression:

$$
N+R\left(\lim _{\pi \rightarrow 0} \frac{d s}{d P}\right)=-\frac{1}{R \beta Q}\left[1+\left(\lim _{\pi \rightarrow 0} \frac{d s}{d P}\right)\right],
$$

Note that $N+R\left(\lim _{\pi \rightarrow 0} \frac{d s}{d P}\right)<0$ since $\lim _{\pi \rightarrow 0} \frac{d s}{d P}>-1$ when $R>N$ (see equation (A.1)). Moreover,

$$
\frac{1}{R \beta Q}\left[1+\left(\lim _{\pi \rightarrow 0} \frac{d s}{d P}\right)\right]>1+\left(\lim _{\pi \rightarrow 0} \frac{d s}{d P}\right)
$$

since $R \beta Q<1$. Therefore, from $(A .10)$ and $(A .11)$, it follows that

$$
N+R\left(\lim _{\pi \rightarrow 0} \frac{d s}{d P}\right)<-\left[1+\left(\lim _{\pi \rightarrow 0} \frac{d s}{d P}\right)\right] .
$$

If we apply inequality $(A .12)$ to the numerator of $\lim _{\pi \rightarrow 0} \frac{d b^{\prime}}{d P}$, which was obtained in $(A .7)$, we get

$$
R^{2} \beta Q^{\prime}-R \beta N Q^{\prime}+\left(1+R^{2} \beta Q^{\prime}\right)\left[N+R\left(\lim _{\pi \rightarrow 0} \frac{d s}{d P}\right)\right]=
$$




$$
\begin{gathered}
R \beta Q^{\prime}(R-N)+N+R\left(\lim _{\pi \rightarrow 0} \frac{d s}{d P}\right)+R^{2} \beta Q^{\prime}\left[N+R\left(\lim _{\pi \rightarrow 0} \frac{d s}{d P}\right)\right]< \\
R \beta Q^{\prime}(R-N)+N+R\left(\lim _{\pi \rightarrow 0} \frac{d s}{d P}\right)-R^{2} \beta Q^{\prime}\left[1+\left(\lim _{\pi \rightarrow 0} \frac{d s}{d P}\right)\right]= \\
-R \beta Q^{\prime}\left[N+R\left(\lim _{\pi \rightarrow 0} \frac{d s}{d P}\right)\right]+N+R\left(\lim _{\pi \rightarrow 0} \frac{d s}{d P}\right)= \\
{\left[N+R\left(\lim _{\pi \rightarrow 0} \frac{d s}{d P}\right)\right]\left(1-R \beta Q^{\prime}\right)<0,}
\end{gathered}
$$

where the last inequality follows from $R \beta Q^{\prime}<1$ and $N+R\left(\lim _{\pi \rightarrow 0} \frac{d s}{d P}\right)<0$.

To prove part (ii) we just have to follow the same arguments as in part (i) and notice that in this case the relevant inequalities are $R \beta Q>1$ and $\lim _{\pi \rightarrow 0} \frac{d s}{d P}<-1$ so that inequalities $(A .11)$ and $(A .12)$ still hold. However, in this case we have that

$$
N+R\left(\lim _{\pi \rightarrow 0} \frac{d s}{d P}\right)>0
$$

as can be seen from equation $(A .10)$ and the fact that now $\lim _{\pi \rightarrow 0} \frac{d s}{d P}<-1$. Finally, combining inequality $(A .14)$ with $R \beta Q^{\prime}>1$, we obtain that inequality $(A .13)$ also holds in this case. 


\section{References}

[1] Abel, A. (1985), "Precautionary Savings and Accidental Bequests", American Economic Review 75, 777-791.

[2] Arrow, K. (1970), Essays in the Theory of Risk Bearing, North Holland, Amsterdam.

[3] Atkinson, A. B. (1980), "Inheritance and Redistribution of Wealth", in Public Policy and the Tax System, edited by G. M. Heal and G. A. Hughes. London: Allen \& Unwin.

[4] Barro, R. (1974), “Are Government Bonds Net Wealth?” Journal of Political Economy 82, 1095-1117.

[5] Becker, G. S. and N. Tomes (1979), "An Equilibrium Theory of the Distribution of Income and Intergenerational Mobility", Journal of Political Economy 87, $1153-1189$.

[6] Bernheim, B.D., A. Schleifer and L.H. Summers (1985), "The Strategic Bequest Motive", Journal of Political Economy 93, 1045-1075.

[7] Bevan, D. L. and J. E. Stiglitz. (1979), "Intergenerational Transfers and Inequality", Greek Economic Review 1, 8-26.

[8] Davies, J. B. (1986), "Does Redistribution Reduce Inequality?", Journal of Labor Economics, 5, 308-335.

[9] Dutta, J. and P. Michel (1998). "The Distribution of Wealth with Imperfect Altruism", Journal of Economic Theory 82, 379-404.

[10] Eckstein, Z, M.S. Eichenbaum, and D. Peled (1985), "Uncertain Lifetimes and the Welfare Enhancing Properties of Annuity Markets and Social Security", Journal of Public Economics 26, 303-326. 
[11] Escolano, J. (1992), Essays on Fiscal Policy, Intergenerational Transfers and the Distribution of Wealth, $\mathrm{Ph}$. D. Dissertation, University of Minnesota

[12] Hadar, J. and R. Russell (1969), "Rules for Ordering Uncertain Prospects", American Economic Review 59, 25-34.

[13] Karni, E and I. Zilcha (1989), "Aggregate and Distributional Effects of Fair Social Security", Journal of Public Economics 40, 37-56.

[14] Kotlikoff, L. and L. Summers (1981), "The Role of Intergenerational Transfers in Aggregate Capital Accumulation", Journal of Political Economy 89, 706-732.

[15] Krusell, P. and A. Smith (1998), "Income and Wealth Heterogeneity in the Macroeconomy", Journal of Political Economy 106, 867-897.

[16] Michel, P. and P. Pestieau (1998), "Fiscal Policy in a Growth Model with Both Altruistic and Nonaltruistic Agents", Southern Economic Journal 64, 682-697.

[17] Pratt, J.W. (1964), "Risk Aversion in the Small and in the Large", Econometrica $32,122-136$.

[18] Sheshinsky E. and Y. Weiss (1981), "Uncertainty and Optimal Social Security Systems", Quarterly Journal of Economics 96, 189-206.

[19] Stokey, N. and R. Lucas with E. C. Prescott (1989), Recursive Methods in Economic Dynamics, Harvard University Press, Cambridge, Mass.

[20] Weil, P. (1987), "Love Thy Children. Reflections on the Barro Debt Neutrality Theorem." Journal of Monetary Economics 19, 377-391.

[21] Yaari, M.E. (1965), "Uncertain Lifetime, Life Insurance, and the Theory of the Consumer", Review of Economic Studies 32, 137-150. 